\title{
Joint Reconstruction of Image and Motion in MRI: Implicit Regularization Using an Adaptive 3D Mesh
}

\author{
Anne Menini ${ }^{1,2}$, Pierre-André Vuissoz ${ }^{1,2}$, \\ Jacques Felblinger ${ }^{1,2}$, and Freddy Odille ${ }^{2}$ \\ 1 IADI, Université de Lorraine, Nancy, France \\ 2 U947, INSERM, Nancy, France \\ \{a.menini,pa.vuissoz,j.felblinger\}@chu-nancy.fr, freddy.odille@inserm.fr
}

\begin{abstract}
Magnetic resonance images are affected by motion artefacts due to breathing and cardiac beating that occur during the acquisition. Methods for joint reconstruction of image and motion have been proposed recently. Such optimization problems are ill-conditioned, therefore regularization methods are required such as motion smoothness constraints using the Tikhonov method. However with Tikhonov methods the solution often relies on a good choice of the regularization parameter $\mu$, especially in large parameter search spaces (e.g. in 3D reconstructions). In this paper, we propose an adaptive, implicit regularization method which results in subject-specific, spatially varying smoothness constraints on the motion model. It is based on the idea of solving for motion only in certain key points that form a mesh. A practical algorithm is proposed for generating this mesh automatically. The proposed method is shown to have a better convergence rate than the Tikhonov method, both in silico and in vivo. The accuracy of the reconstructed image and motion is also improved.
\end{abstract}

Keywords: Magnetic Resonance Imaging, reconstruction, non-rigid motion, regularization, mesh, inverse problem.

\section{Introduction}

A magnetic resonance imaging (MRI) acquisition is a sequential process and therefore often exceeds tolerable breath hold time. Thus, respiratory motion and heart beating can occur during the acquisition. Since data are acquired in the Fourier domain, such non-rigid motions lead to complex artefacts that affect the whole image. These cannot be fixed using entirely image post-processing methods such as registration. Solutions cannot be found among acquisition strategies either. Indeed, despite effective acceleration methods [1, motion correction remains a challenge because of the growing interest in $3 \mathrm{D}$ and $4 \mathrm{D}(3 \mathrm{D}+$ time $)$, high resolution imaging and quantitative MRI which take minutes to acquire.

Reconstruction methods have been proposed based on the joint optimization of image and motion [2, 3]. The two unknowns obtained in this way are an 
artefact-free image and a non-rigid motion model that describes the displacements of each voxel during the acquisition. This leads to a non-linear optimization which is generally ill-conditioned. Therefore, a regularization is applied to constrain the solution. A typical constraint consists of picking up the solution whose motion field is smoothest, as defined by the gradient $\mathcal{L}_{2}$ norm, which is one kind of Tikhonov regularization.

Such Tikhonov methods have several drawbacks : i) the weight of the smoothness constraint, as expressed by a regularization parameter called $\mu$ in the remainder of the paper, is generally adjusted empirically (optimizing $\mu$ itself would necessitate a considerable increase in reconstruction time) ; ii) such regularizers may not depict certain classes of motion, e.g. shearing or piecewise smooth motion ; iii) when the dimensionality of the problem is increased (e.g. from $2 \mathrm{D}$ to $3 \mathrm{D})$, the conditioning of the problem decreases and thus leads to a higher sensitivity of the solution to $\mu$, both in terms of accuracy and convergence rate.

In this paper, we propose an alternative regularization method intended to avoid these drawbacks. Instead of dealing with a large amount of unknowns and imposing a strong constraint, here we solve the problem only for a small subset of unknowns that can be thought of as describing the principal components of motion. The method proceeds by generating an adaptive mesh which inherently contains local smoothness information. Once integrated into the joint reconstruction process, it allows voxels with similar displacements to be grouped and thereby provides an implicit regularization for the optimization of the motion model, meaning the explicit regularization term is no longer necessary.

The proposed method, the adaptive regularization (AR), was compared with a classical Tikhonov regularization (TR), both in silico and in vivo. Results were assessed in term of convergence rate, image similarity and motion recovery.

\section{Joint Reconstruction of Image and Motion}

\subsection{Inverse Problem}

We are interested in reconstructing a volume (or an image) $\rho_{0}$ that was acquired while an unknown non-rigid motion occurs. This can be described by a set of images $\left(\rho_{t}\right)_{t=1 . . N_{t}}$ at several physiological states,

$$
\rho_{t}=W_{t}\left(u_{t}\right) \rho_{0}
$$

where $W_{t}$ is an image transformation matrix that depends on an unknown displacement $u_{t}$. The displacement $u_{t}$ can be parametrized, e.g. using physiological signals $\left(S_{k}\right)_{k=1 . . N_{k}}$ from motion sensor that are temporally correlated with physiological movements (e.g. abdominal or thoracic breathing, heart beating). Then the displacement can be approximated by a linear combination

$$
u_{t}(r)=\sum_{k=1}^{N_{k}} \alpha_{k}(r) S_{k}(t)
$$


where $\left(\alpha_{k}\right)_{k=1 . . N_{k}}$ are unknown motion models coefficients. The warping of the image is rewritten as a function of the unknown motion parameters.

$$
\rho_{t}=W_{t}(\alpha) \rho_{0}
$$

The acquisition process can be expressed by a linear forward projection operator $P$. Then, the raw data $s$ acquired by the scanner are given by an encoding operator $E(\alpha)$ affected by noise $\nu$.

$$
s=\sum_{t=1}^{N_{t}} P_{t} W_{t}(\alpha) \rho_{0}+\nu=E(\alpha) \rho_{0}+\nu
$$

Eventually, the joint reconstruction problem consists in solving the inverse problem of (4). Generally, it is done by minimizing the negative log likelihood of:

$$
L\left(\rho_{0}, \alpha\right)=\left\|s-E(\alpha) \rho_{0}\right\|^{2} .
$$

\subsection{Joint Optimization with Explicit Regularization}

While the problem of reconstructing $\rho_{0}$ for a fixed motion model $\alpha$ reduces to a well conditioned least squares problem, the problem of recovering both $\rho_{0}$ and $\alpha$ is ill-posed. This is because several motion models can lead to the same warped image $\rho_{t}$. A regularization is applied to constrain the solution, for example to be smooth or to have a low energy. It is achieved by adding a regularization operator.

$$
\left(\tilde{\rho_{0}}, \tilde{\alpha}\right)=\underset{\rho_{0}, \alpha}{\operatorname{argmin}}\left\{L\left(\rho_{0}, \alpha\right)+\Psi(\alpha)\right\}
$$

With a Tikhonov regularization, we can write $\Psi(\alpha)=\mu\|\nabla \alpha\|_{2}^{2}$. Equation (6) can be solved by alternating optimization with respect to $\rho_{0}$ and $\alpha$. Two linearised problems are solved alternately using standard iterative linear system solvers. A multi-resolution scheme can also be used as no initial guess of the motion model $\alpha$ is available in general.

We consider an acquisition with $N_{k}$ motion models like in (2). In 2D, the displacements can only be described in-plane (2 directions), so we obtain $N_{\alpha, 2 D}$ unknowns for the motion with $N_{\alpha, 2 D}=2 \times N_{k} \times N_{x} \times N_{y}$ and $N_{s, 2 D}$ data acquired with $N_{s, 2 D}=N_{x} \times N_{y}$. Then in 3D, we obtain $N_{\alpha, 3 D}=3 \times N_{k} \times N_{x} \times N_{y} \times N_{z}$ and $N_{s, 3 D}=N_{x} \times N_{y} \times N_{z}$. The amount of unknowns raises $3 / 2$ times more than the amount of data. Consequently, the inverse problem is much more illconditioned in 3D. To tackle that issue the constraint should have a stronger weight, however this can result in excessive smoothing.

\section{Implicit Regularization}

\subsection{Assumptions about Motion}

Regularizations are often based on the assumption that motion is relatively smooth. While this is a reasonable assumption within a single tissue, it is invalidated at the interface between different tissues moving in independent directions (e.g. shearing between liver and ribs). 
Then, it would be more efficient to allow local variations of the constraint rather than applying the same constraint to the whole image. This can be achieved by varying the density of points where the motion is described (referred as key points). By doing so, motion is constrained to be smooth only where the density of key points is low (Fig. 11). Furthermore, it reduces drastically the number of unknowns which makes the optimization task more tractable.
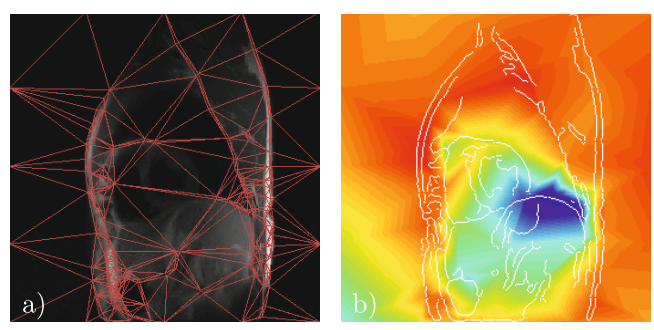

Fig. 1. Example of a 2D mesh adapted for a short axis MRI acquisition (a). The motion model reconstructed is smooth where the density of the mesh is low (b)

\subsection{Mesh Generation}

We suppose that we have a first estimate of the image $\rho$ and the motion $\alpha$. These can be provided by a previous iteration of the joint reconstruction algorithm. We propose the algorithm 1 to select the set of points $\Omega$ where the motion should be solved for.

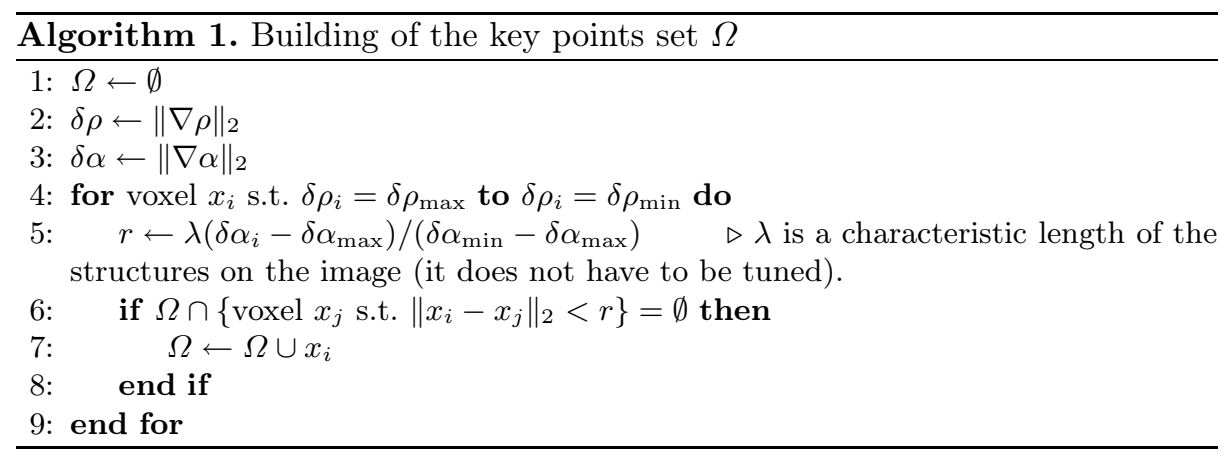

This mesh generation algorithm results in selecting with higher priority points that lie at the interfaces between different tissues (corresponding to large image gradients). Moreover, the density of points is proportional to the complexity of the motion (reflected in the motion model gradients). To initialize the algorithm when no motion model estimate is available, vertices are simply spread along the image gradients. With this point set, voxels with similar displacements are 
automatically grouped which has the effect of a spatially varying regularization. In addition, we avoid the pitfalls of classical regularizations. More particularly, the selection of the key point set is automatic (no parameter tuning). The mesh generation should be relatively fast for the joint reconstruction algorithm to benefit from it. Hence this direct method is preferable to iterative ones.

\subsection{Mesh Integration}

The image can be split into pieces with a Delaunay tesselation of the key points set [4. Thus, a mesh is obtained. In $2 \mathrm{D}$ (resp. 3D), each point $P_{i}$ in space belongs a triangle $T_{i}$ (resp. tetrahedron). Thereby, its motion depends on the unknown motion $\left(\hat{\alpha}_{T_{i}, j}\right)_{j=1 . . N_{V}}$ of only $N_{V}$ vertices $\left(V_{T_{i}, j}\right)_{j=1 . . N_{V}}$ with $N_{V}=3$ (resp. $\left.N_{V}=4\right)$. Then, the motion $\alpha_{i}$ of a point $P_{i}$ in space can be obtained by a barycentric interpolation.

$$
\left\{\begin{array}{l}
P_{i}=\sum_{j=1}^{N_{V}} \beta_{i j} V_{T_{i}, j} \\
\alpha_{i}=\sum_{j=1}^{N_{V}} \beta_{i j} \hat{\alpha}_{T_{i}, j}
\end{array}\right.
$$

As a consequence of using a mesh, there is no need to compute the motion explicitly in every point to warp an image. Indeed, we can compute a texture mapping based on the mesh. It can be achieved with a piece-wise affine interpolation (Algo. 2 and Fig. 2).
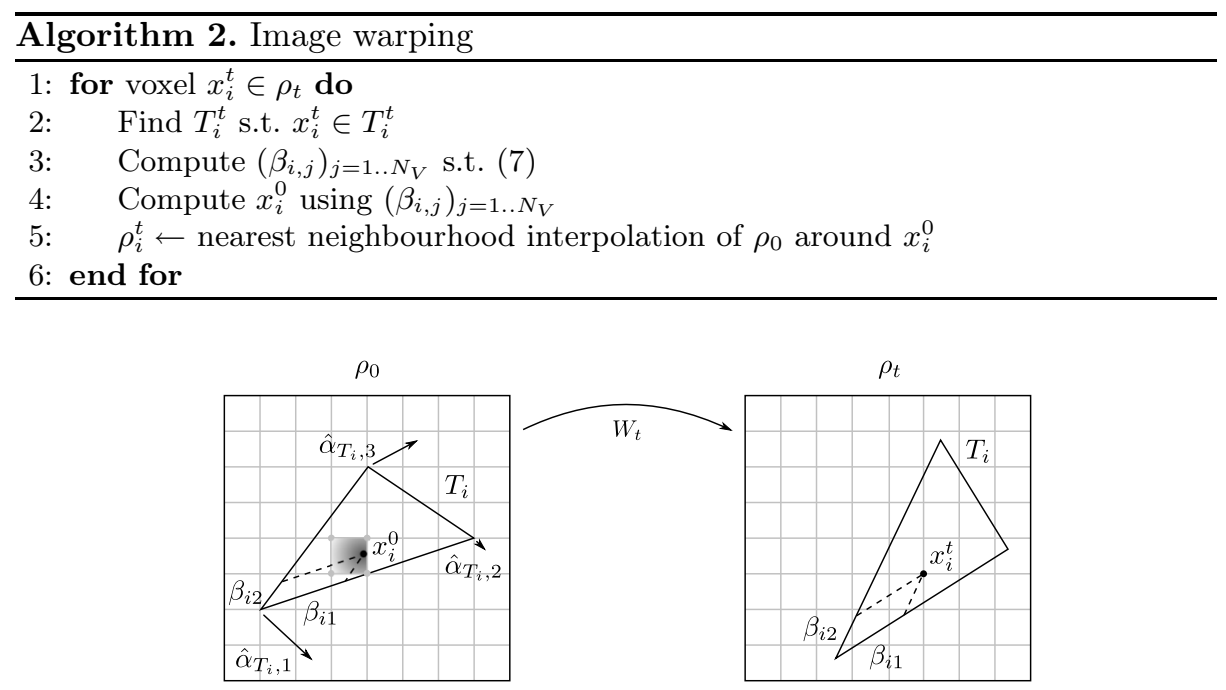

Fig. 2. Image warping with a piece-wise affine interpolation

The mesh could be updated each time the image or motion parameters are updated. Here, in a multiresolution reconstruction, we suggest to update the mesh only once at the beginning of each resolution loop. 


\section{Validation}

\subsection{In Silico}

A 3D MRI acquisition was simulated from a synthetic image (Fig. 3 (a)), a predefined motion model (Fig. 3 (b, c, d)) and real physiological signals according to (2. 4). This data set simulate a sagittal view of a liver through a 3D MRI sequence with parallel imaging. Two joint reconstructions of the image and the motion models were performed with a fixed-point multi-resolution scheme [2], one with the classical Tikhonov method and the other with our adaptive method to regularize the motion optimization. Several reconstructions were tested with the Tikhonov method in order to find a fair regularization parameter $(\mu=0.01)$.
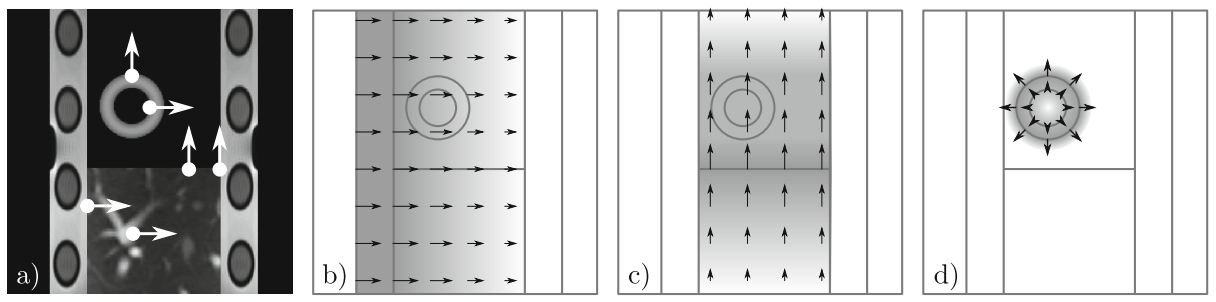

Fig. 3. Synthetic image with locations where the motion accuracy was assessed (a), and motion models used to simulate the data: thoracic breathing (b), abdominal breathing (c) and cardiac beating (d)
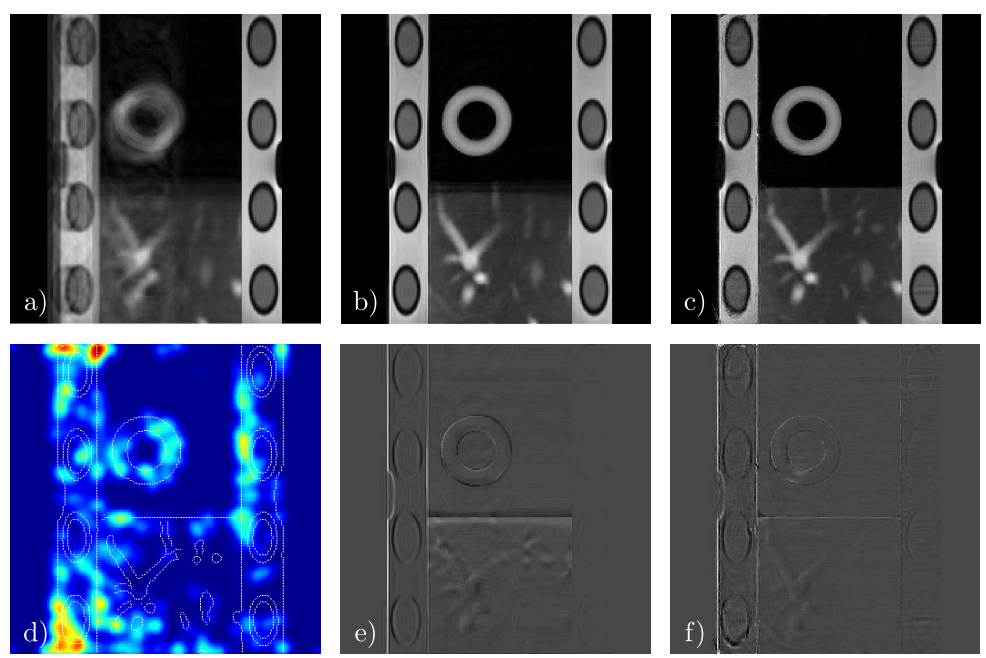

Fig. 4. Reconstructions of the synthetic data set: without motion correction (a), with the motion correction using TR (b), using AR (c). Point density of the adaptive mesh (d). Error with TR (e) and error with AR (f). 
The image obtained with the naive Fourier reconstruction presents motion artefacts as expected (Fig. 4 (a)). Both regularizations have successfully solved for the thoracic breathing and the cardiac beating (Fig. 4 (b, c)). However, motion artefacts from abdominal breathing remain with the classical regularization. Since the latter motion model includes abrupt variations unlike the other ones, it points out the limitation of a homogeneous smoothing constraint. The NRMSE computed on the image reaches $5.3 \%$ with the classical method, against $4.7 \%$ with the adaptive one (Fig. 4 (e, f)). Similarly, the NRMSE computed on 6 specific locations of the motion reaches $69.5 \%$, against $22.3 \%$ (Fig. 3 (a)). The amount of unknowns for the motion was divided by 100 with the adaptive method. At last, for each resolution level, the number of iterations $n$ required for the optimization to converge to the same given tolerance systematically reached the maximum number allowed $\left(n_{\max }=8\right)$ with the Tikhonov regularization whereas it reached 3.3 in average with our method.

\subsection{In Vivo}

3D MRI acquisitions 1 of the liver were performed in a healthy volunteer during free breathing and during breath hold. Similarly, two joint reconstructions were performed from the free breathing acquisitions. Qualitatively, both regularization methods resulted in a drastic improvement of the image compared to uncorrected images, and were similar to the breath hold acquisition (Fig. [5). The convergence behaviour was better for the adaptive method like in silico $(n=3$ against 8$)$.
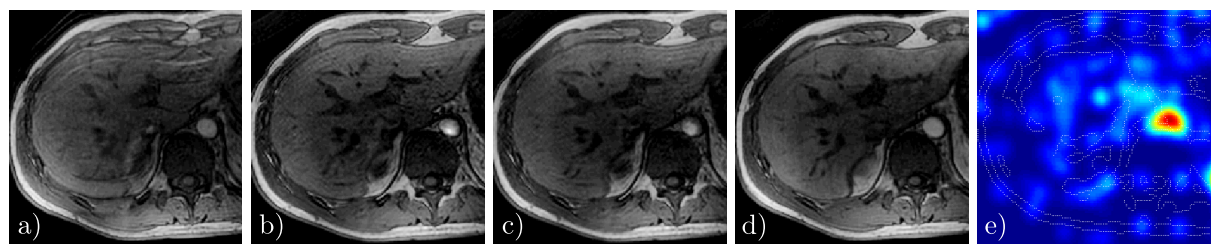

Fig. 5. Reconstructions of the liver on a healthy volunteer (zoom): free breathing acquisition without motion correction (a), with the motion correction using TR (b), using AR (c) and acquisition in breath hold (d). Point density of the adaptive mesh (e).

\section{Discussion}

Finally, our method makes the regularization of highly ill-conditioned problems more tractable. Therefore, compared with the Tikhonov method, it improves the accuracy of the joint optimization solutions and in addition, speeds up the convergence rate and reduces the number of unknowns by 100 .

Here we chose to report the convergence rate (number of iteration), rather than the effective computation time. Indeed, our new method involves several

${ }^{1}$ LAVA, $T E / T R=1.82 / 3.92 \mathrm{~ms}$, matrix size: $320 \times 288 \times 60$, asset factor $=2,3 \mathrm{~T}$ SIGNA HDxt, GE Healthcare, Milwaukee, WI. 
time consuming algorithms (e.g. Delaunay tesselation, search for the triangle containing some point, interpolation), which have not been optimized yet in our current implementation. Till then, these loss in computation time are compensated by the drop-off of iterations required. A GPU implementation of these steps should improve the computation time as well.

Alternative regularizers have been proposed in the literature to address the limitations of Tikhonov regularizers. In particular, total variation regularization [5] allows discontinuities to be preserved. However this is at the cost of an increased complexity of the optimization leading to increased computation time.

Finite element approaches are often used [6]; however these are not always adaptive. Others have proposed adaptive meshes [7. Their adaptation consists of an iterative refinement based on residual errors at the previous iteration. In our case, the construction is direct when we have a motion estimate, which is available after only one iteration. These adaptive techniques could be combined.

The adaptive mesh-based implicit regularization is not limited to free-breathing MRI reconstruction. Image registration techniques [8] might also apply.

\section{References}

[1] Tsao, J.: Ultrafast imaging: Principles, pitfalls, solutions, and applications. Journal of Magnetic Resonance Imaging 32(2), 252-266 (2010)

[2] Odille, F., Vuissoz, P., Marie, P., Felblinger, J.: Generalized reconstruction by inversion of coupled systems (GRICS) applied to free-breathing MRI. Magnetic Resonance in Medicine 60(1), 146-157 (2008)

[3] Jacobson, M.W., Fessler, J.A.: Joint estimation of image and deformation parameters in motion-corrected PET. In: 2003 IEEE Nuclear Science Symposium Conference Record, vol. 5, pp. 3290-3294. IEEE (October 2003)

[4] Barber, C.B., Dobkin, D.P., Huhdanpaa, H.: The quickhull algorithm for convex hulls. ACM Transaction on Mathematical Software 22(4), 469-483 (1996)

[5] Rudin, L.I., Osher, S., Fatemi, E.: Nonlinear total variation based noise removal algorithms. Physica D: Nonlinear Phenomena 60(14), 259-268 (1992)

[6] Arridge, S.R., Schweiger, M., Hiraoka, M., Delpy, D.T., et al.: A finite element approach for modeling photon transport in tissue. Medical Physics-Lancaster PA 20, 299-299 (1993)

[7] Joshi, A., Bangerth, W., Sevick-Muraca, E.: Adaptive finite element based tomography for fluorescence optical imaging in tissue. Optics Express 12(22), 5402-5417 (2004)

[8] Rueckert, D., Sonoda, L.I., Hayes, C., Hill, D.L., Leach, M.O., Hawkes, D.J.: Nonrigid registration using free-form deformations: application to breast $\mathrm{MR}$ images. IEEE Transactions on Medical Imaging 18(8), 712-721 (1999) 